Presented at the $11^{\text {th }}$ International Conference on Ion Beam Modification of Materials, Amsterdam, 31.8.-4.9.1998

\title{
Lattice sites and damage annealing of Er in low-dose implanted GaAs
}

\author{
U. Wahl, A. Vantomme, G. Langouche, Instituut voor Kern- en Stralingsfysica, \\ University of Leuven, Celestijnenlaan 200 D, B-3001 Leuven, Belgium \\ The ISOLDE collaboration, CERN-PPE, CH-1211 Genève 23, Switzerland
}

\begin{abstract}
We have used conversion electron emission channeling to determine the lattice location of ${ }^{167 \mathrm{~m}} \operatorname{Er}\left(\mathrm{t}_{1 / 2}=2.28 \mathrm{~s}\right)$ in GaAs after $60 \mathrm{keV}$ room temperature implantation of ${ }^{167} \mathrm{Tm}\left(\mathrm{t}_{1 / 2}=9.25 \mathrm{~d}\right)$ at low doses $\left(0.6-3 \times 10^{13} \mathrm{~cm}^{-2}\right)$. Following a recovery step of the implantation damage at $200-300^{\circ} \mathrm{C}$, we observe a large fraction of $\operatorname{Er}(45-68 \%)$ on substitutional Ga sites. A second fraction of $\mathrm{Er}$ is found on the $\mathrm{T}_{\mathrm{As}}$ sites (the tetrahedral interstitial sites with nearest As neighbours). The fraction on $\mathrm{T}_{\mathrm{As}}$ sites reaches a maximum of 12-23\% following annealing at 500$600^{\circ} \mathrm{C}$. At higher annealing temperatures the channeling effects decrease markedly, which we attribute to the well-known degradation of GaAs due to As evaporation.
\end{abstract}

PACS codes: 61.72.-y, 61.72.Vv, 61.85.+p

Keywords: lattice location, emission channeling, GaAs, erbium, ion implantation

Ulrich Wahl

Instituut voor Kern- en Stralingsfysica, Katholieke Universiteit Leuven, Celestijnenlaan 200 D, B-3001 Leuven, Belgium

e-mail: ulrich.wahl@fys.kuleuven.ac.be, phone: ++32-16-327617, fax: ++32-16-327985

\section{Introduction}

Erbium-doped semiconductors are of considerable interest as optoelectronic devices [1]. In general, the optical activity of $\mathrm{Er}$ is closely related to its lattice site and immediate surroundings. For Er in GaAs, theoretical investigations suggest that isolated Er prefers substitutional $\mathrm{Ga}$ sites $\left(\mathrm{S}_{\mathrm{Ga}}\right)$ and that even the interaction with additional native defects such as $\mathrm{Ga}_{\mathrm{i}}, \mathrm{As}_{\mathrm{i}}$, or $\mathrm{V}_{\mathrm{As}}$ is hardly able to remove it from this site [2]. Er on tetrahedral interstitial sites $(\mathrm{T})$ is considered to be metastable, and the $\mathrm{T}_{\mathrm{As}}$ sites should be lower in energy than the $\mathrm{T}_{\mathrm{Ga}}$ sites.

On the experimental side, a number of Rutherford backscattering (RBS) lattice location studies on GaAs:Er revealed the following trends. In general, samples doped with Er during metalorganic chemical vapour deposition (MOCVD) [3-6] or molecular beam epitaxy (MBE) [7-9] showed the majority of Er in neartetrahedral interstitial locations with respect to the GaAs lattice. A discrimination between $\mathrm{T}_{\mathrm{As}}$ and $\mathrm{T}_{\mathrm{Ga}}$ sites, however, was not considered in any of these studies. Er concentrations in these samples varied in a wide range from $10^{17} \mathrm{~cm}^{-3}$ to $7 \times 10^{20} \mathrm{~cm}^{-3}$. The interstitial-like Er location in the high-dose regime was related to the formation of ErAs precipitates growing epitaxially along the GaAs <100> directions. In the low-dose doped samples, on the other hand, the interstitial position was ascribed to complexes between Er and carbon, which is a frequent contaminant in MOCVD. Samples which were intentionally co-doped with oxygen during MOCVD growth, however, showed mainly near-substitutional Er [6, 10].

Less studies have dealt with the lattice location of implanted Er in GaAs. Following high-dose implantation at $250^{\circ} \mathrm{C}\left(10^{15} \mathrm{~cm}^{-2}\right.$ at $150 \mathrm{keV}$, peak concentration of $\left.2.8 \times 10^{20} \mathrm{~cm}^{-3}\right)$ and annealing up to $1000^{\circ} \mathrm{C}, \mathrm{Er}$ atoms were found within the $\langle 100\rangle$ but partly outside the $\left.<110\right\rangle$ atomic rows [11, 12]. While such a behaviour could be due to a mixture of substitutional (S) and tetrahedral interstitial (T) Er, it would also be expected for Er within large $<100\rangle$ epitaxial ErAs precipitates. Only the outermost Er atoms in the precipitates sense the particle flux-peaking within the $\langle 110\rangle$ channel, at the same time they shadow the Er atoms in deeper ErAs layers [10]. Recently the Er sites following implantations at lower doses $\left(0.75-7.5 \times 10^{14} \mathrm{~cm}^{-2}, 100-250\right.$ $\mathrm{keV}, \approx 10^{19}-10^{20} \mathrm{~cm}^{-3}$ ) and annealing at $850^{\circ} \mathrm{C}$ were investigated [13]. A $7.5 \times 10^{13} \mathrm{~cm}^{-2}$ implantation resulted in $80 \%$ of Er on S, $5 \%$ on $\mathrm{T}$ and $15 \%$ on random sites, while higher doses yielded only $10 \%$ on $\mathrm{S}$, but $55 \%$ on $\mathrm{T}$ and $35 \%$ on random sites. In this work the interstitial fraction was ascribed to small ErAs precipitates.

In order to extend the Er lattice location studies to even lower doses, we have applied conversion electron emission channeling [14] from the short-lived isotope ${ }^{167 \mathrm{~m}} \operatorname{Er}\left(\mathrm{t}_{1 / 2}=2.28 \mathrm{~s}\right)$. This excited nuclear state is 
populated as a consequence of the radioactive decay of the isotope ${ }^{167} \mathrm{Tm}(9.25 \mathrm{~d})$ and deexcites into the ${ }^{167} \mathrm{Er}$ ground state by the emission of conversion electrons. The emitted electrons experience channeling or blocking effects along low-index crystal directions. This leads to an anisotropic emission yield from the crystal surface which depends in a characteristic way on the lattice sites occupied by the emitter atoms. Previously we have already used this method to study the lattice sites of Er in $\mathrm{Si}[15,16]$.

\section{Experimental}

The samples used in our experiments were two $<100>$ cut n-GaAs:Te single crystals $\left(0.6-2 \times 10^{-3} \Omega \mathrm{cm}, n=1\right.$ $5 \times 10^{18} \mathrm{~cm}^{-3}$ ). The radioactive isotope ${ }^{167} \mathrm{Tm}$ was implanted at $60 \mathrm{keV}$ by means of the ISOLDE facility at CERN, using a $1 \mathrm{~mm}$ beam spot, $7^{\circ}$ tilted implantation and doses of $6 \times 10^{12} \mathrm{~cm}^{-2}$ (sample A) and $3 \times 10^{13} \mathrm{~cm}^{-2}$ (sample B). These parameters correspond to [Tm+Er] peak concentrations of $3 \times 10^{18} \mathrm{~cm}^{-3}$ (A) and $1.5 \times 10^{19} \mathrm{~cm}^{-}$ ${ }^{3}$ (B) at a depth of $198 \AA$ (FWHM $78 \AA$ ). Following the room temperature implantations the samples were annealed capless in vacuum $<10^{-5}$ mbar. In order to detect the angular-dependent emission yield of the 150,199 and $206 \mathrm{keV}$ conversion electrons emitted by ${ }^{167 \mathrm{~m}} \mathrm{Er}$ we used the position- and energy sensitive detection system described in Ref. [16].

\section{Results and discussion}

Fig. 1(a)-(c) show the normalized ${ }^{167 \mathrm{~m}}$ Er conversion electron emission yields around the $\langle 111\rangle,\langle 100\rangle$ and $<110\rangle$ directions of sample A following the annealing sequence to $300^{\circ} \mathrm{C}$. Pronounced emission channeling effects are visible along all axial and planar directions, which is clear evidence that the majority of ${ }^{167 \mathrm{~m}} \mathrm{Er}$ occupies substitutional sites. In order to analyze the lattice location more precisely we fitted theoretical emission patterns for different sites to the experimental data. The fitting procedures applied for that purpose are described in detail in Ref. [16]. The theoretical emission patterns were calculated using the "many beam" approach of electron motion in single crystals [14].

Extracting reliable lattice location information for the III-V compound GaAs requires to consider the characteristic properties of the underlying zincblende structure [17]. For instance, the <111> patterns do not allow to discriminate between $\mathrm{S}$ and $\mathrm{T}$ sites; in addition both the Ga and the As sublattice show the same projection along this direction. The best fit to the <111> pattern [Fig. 1(d)] was obtained for $85 \%$ of emitter atoms on $(\mathrm{S}+\mathrm{T})$ sites with a root mean square (rms) displacement of $\mathrm{u}_{1}=0.2 \AA$ and $15 \%$ on random (R) sites. Along $\left\langle 100>\right.$ directions $S_{G a}$ and $T_{A s}$ sites lie within pure Ga rows while $S_{A s}$ and $T_{G a}$ are located within pure As rows. By $\mathrm{T}_{\mathrm{As}}$ we denote those $\mathrm{T}$ sites which have 4 nearest As neighbours in $\langle 111\rangle$ and 6 next-nearest Ga neighbours in $\langle 100\rangle$ directions. In principle the $\langle 100\rangle$ axis could therefore allow to discriminate between $\left(\mathrm{S}_{\mathrm{Ga}}+\mathrm{T}_{\mathrm{As}}\right)$ and $\left(\mathrm{S}_{\mathrm{As}}+\mathrm{T}_{\mathrm{Ga}}\right)$. However, the nuclear charges of $\mathrm{Ga}$ and As are so similar that in practice it is impossible to distinguish the $<100\rangle \mathrm{Ga}$ and As rows reliably. The best fit [Fig. 1(e)] was obtained for $82 \%$ on $(\mathrm{S}+\mathrm{T})$ with $\mathrm{u}_{1}=0.16 \AA$, and $18 \%$ on $\mathrm{R}$ sites. Finally, the $<110>$ patterns allow to distinguish between all various interstitial sites and between both sublattices. Rather than being a consequence of the different nuclear charges of the $\langle 110\rangle \mathrm{Ga}$ or As rows, the sublattice sensitivity is a structural effect related to the mirror-asymmetry of the $\left\langle 110>\right.$ directions of the GaAs lattice. Hence Er preferring sites in one specific sublattice, e.g., $\mathrm{S}_{\mathrm{Ga}}$, causes an asymmetry in the <110> patterns. On the other hand, if the Er would be equally distributed among both sublattices, the electron yield around the $\langle 110\rangle$ direction should be almost symmetric with respect to mirroring at the $\{100\}$ plane, which is not the case [Fig. 1(c)]. Best fits [Fig. 1(f)] were obtained for $65 \%$ of Er on $\mathrm{S}_{\mathrm{Ga}}$ with $\mathrm{u}_{1}=0.16 \AA, 16 \%$ on $\mathrm{T}_{\mathrm{As}}$, and $19 \%$ on random sites. Excluding the $\mathrm{T}_{\mathrm{As}}$ sites from the fit resulted in a $15 \%$ worse chi square. Due to the rather small fraction of $\mathrm{Er}$ on $\mathrm{T}_{\mathrm{As}}$, it was not possible to determine the rms displacement from this site reliably. Fits including also $\mathrm{Er}$ on $\mathrm{S}_{\mathrm{As}}$ or $\mathrm{T}_{\mathrm{Ga}}$ resulted in a few percent improvement of the chi square values while the corresponding fractions on both sites were less than $5 \%$, which is about the statistical uncertainty. Including additional lattice sites such as bond centered (BC), anti-bonding (AB), hexagonal $(\mathrm{H})$ or displaced sites also yielded only insignificant improvements in fit quality; possible fractions on these sites are estimated to be below $10 \%$.

Fig. 2 summarizes the results of the annealing sequences for both samples. In both cases clear channeling effects were already visible directly following room temperature implantation, corresponding to around $38 \%$ of $\mathrm{Er}$ on $\mathrm{S}_{\mathrm{Ga}}$ sites in the low-dose sample A, and 13\% in the higher-dose sample B. The remaining Er fraction occupies random sites, i.e. sites in disordered surroundings which are responsible for an isotropic electron emission yield. In sample A the channeling effects improved markedly following annealing at $200^{\circ} \mathrm{C}$, resulting more or less in the Er fractions on $\mathrm{S}_{\mathrm{Ga}}$ and $\mathrm{T}_{\mathrm{As}}$ sites discussed above. On the other hand, in sample $\mathrm{B}$ the recovery step was shifted to higher temperatures and a larger amount of Er (50\%) remained on $\mathrm{R}$ sites. This behaviour indicates incomplete structural recovery of the radiation damage in this higher-dose implanted sample. 
In between the annealing steps at $600^{\circ} \mathrm{C}$ and $650^{\circ} \mathrm{C}$, sample $\mathrm{A}$ was heated again at $500^{\circ} \mathrm{C}$ for $2 \mathrm{~h}$ in order to measure in situ the emission channeling effects at this temperature. The results were essentially the same as measuring at room temperature following the $600^{\circ} \mathrm{C}$ anneal (Fig.2). This shows that the $\mathrm{S}_{\mathrm{Ga}}$ sites are thermally stable during the life time of the excited ${ }^{167 \mathrm{~m}} \mathrm{Er}$ state and not only metastable positions which are populated as a consequence of the ${ }^{167} \mathrm{Tm}$ decay.

In both samples a smaller part of Er was found on the $\mathrm{T}_{\mathrm{As}}$ sites. In sample $\mathrm{A}$ the fraction on $\mathrm{T}_{\mathrm{As}}$ grew with increasing annealing temperature up to $500^{\circ} \mathrm{C}$ on the expense of the $\mathrm{S}_{\mathrm{Ga}}$ sites. Upon annealing to $650^{\circ} \mathrm{C}$, the $\mathrm{T}_{\mathrm{As}}$ fraction decreased to zero, while the substitutional $\mathrm{S}_{\mathrm{Ga}}$ fraction was still around $35 \%$. In sample $\mathrm{B}$, on the other hand, less Er was found on $\mathrm{T}_{\mathrm{As}}$ sites, and the maximum occurred after annealing around $625^{\circ} \mathrm{C}$, when the fraction on $\mathrm{S}_{\mathrm{Ga}}$ sites had already considerably decreased. Er on $\mathrm{T}_{\mathrm{As}}$ sites can not be explained by the formation of epitaxial ErAs precipitates, since the Er atoms at the surface of the precipitates are supposed to be located on $\mathrm{T}_{\mathrm{Ga}}$ sites [13]. Moreover, we would also expect that the probability of forming such clusters and hence the fraction of Er on T-like sites would be higher in the higher dose sample B. The increasing occupation of $\mathrm{T}_{\mathrm{As}}$ sites is therefore not well understood at the moment. The vanishing of the channeling effects following annealing above $600^{\circ} \mathrm{C}$ is not astonishing. It has been reported in the literature that vacuum annealing of GaAs above $500^{\circ} \mathrm{C}$ without As overpressure or capping causes excessive As losses within the first few hundred $\AA$ and sample degradation [18].

\section{Conclusions}

We have extended the earlier results of Alves et al [13], who found the majority of implanted Er in GaAs on substitutional sites, to even lower doses. Moreover, we give direct evidence that, in agreement with theoretical predictions [2], Er prefers the $\mathrm{S}_{\mathrm{Ga}}$ sites. The displacements from these sites were found to be small, of the order of $\mathrm{u}_{1}=0.16-0.20 \AA$ in the case of isotropic displacements such as lattice vibrations, or around $\mathrm{d}=0.20-0.25 \AA$ for static displacements. A second, smaller fraction of $\mathrm{Er}$ was found on $\mathrm{T}_{\mathrm{As}}$ sites, also in agreement with predictions that the $\mathrm{T}_{\mathrm{As}}$ sites should be the most stable interstitial sites for Er. However, more detailed experiments will be required to better understand the thermal behaviour of the fractions of Er on both $\mathrm{S}_{\mathrm{Ga}}$ and $\mathrm{T}_{\mathrm{As}}$ sites.

\section{References}

[1] H. Ennen, J. Schneider, G. Pomrenke, and A. Axmann, Appl. Phys. Lett. 43 (1983) 943.

[2] A. Taguchi and T. Ohno, Phys. Rev. B 56 (1997) 9477.

[3] J. Nakata, M. Taniguchi and K. Takahei, Appl. Phys. Lett. 61 (1992) 2665.

[4] S.J. Chang, K. Takahei, J. Nakata and Y.K. Su, Mat. Res. Soc. Symp. Proc. 301 (1993) 299.

[5] Y. Kido, T. Ishida, E. Nakai, M. Saeki, J. Nakata, and K. Takahei, Nucl. Instr. Meth. B 85 (1994) 484.

[6] J. Kaczanowski, Y. Yamamoto, Y. Kido, J. Nakata and K. Takahei, Nucl. Instr. Meth. B 117 (1996) 275.

[7] E. Alves, M.F. da Silva, K.R. Evans, C.R. Jones, A.A. Melo and J.C. Soares, Nucl. Instr. Meth. B 80/81 (1993) 180.

[8] E. Alves, M.F. da Silva, A.A. Melo, J.C. Soares, G.N. van den Hoven, A. Polman, K.R. Evans and C.R. Jones, Mat. Res. Soc. Symp. Proc. 301 (1993) 175.

[9] Y. Kido, A. Ikeda, Y. Yamamoto, J. Nakata, H. Yamaguchi and K. Takahei, Phys. Rev. B 48 (1994) 14387.

[10] K. Takahei, A. Taguchi, Y. Horikoshi and J. Nakata, J. Appl. Phys. 76 (1994) 4332.

[11] A. Kozanecki, M. Chan, C. Jeynes, B. Sealy and K. Homewood, Sol. State Comm. 78 (1991) 763.

[12] A. Kozanecki, J.M. Langer and A.R. Peaker, Acta Physica Polonica 83 (1993) 59.

[13] E. Alves, M.F. da Silva, J.C. Soares, M.O. Henry, R. Gwilliam, B.J. Sealy, K. Freitag, R. Vianden and D. Stievenard, Nucl. Instr. Meth. B 136-138 (1998) 421.

[14] H. Hofsäss and G. Lindner, Phys. Rep. 201 (1991) 123.

[15] U. Wahl, A. Vantomme, J. De Wachter, R. Moons, G. Langouche, J.G. Marques, J.G. Correia, and the ISOLDE collaboration, Phys. Rev. Lett. 79 (1997) 2069.

[16] U. Wahl, J.G. Correia, S. Cardoso, J.G. Marques, A. Vantomme, G. Langouche and the ISOLDE collaboration, Nucl. Instr. Meth. B 136-138 (1998) 744.

[17] U. Wahl, Phys. Rep. 280 (1997) 145.

[18] S.T. Picraux, in Ion Implantation in Semiconductors and other Materials, ed. B Crowder (Plenum Press, New York ,1973) p. 641. 


\section{Figures}

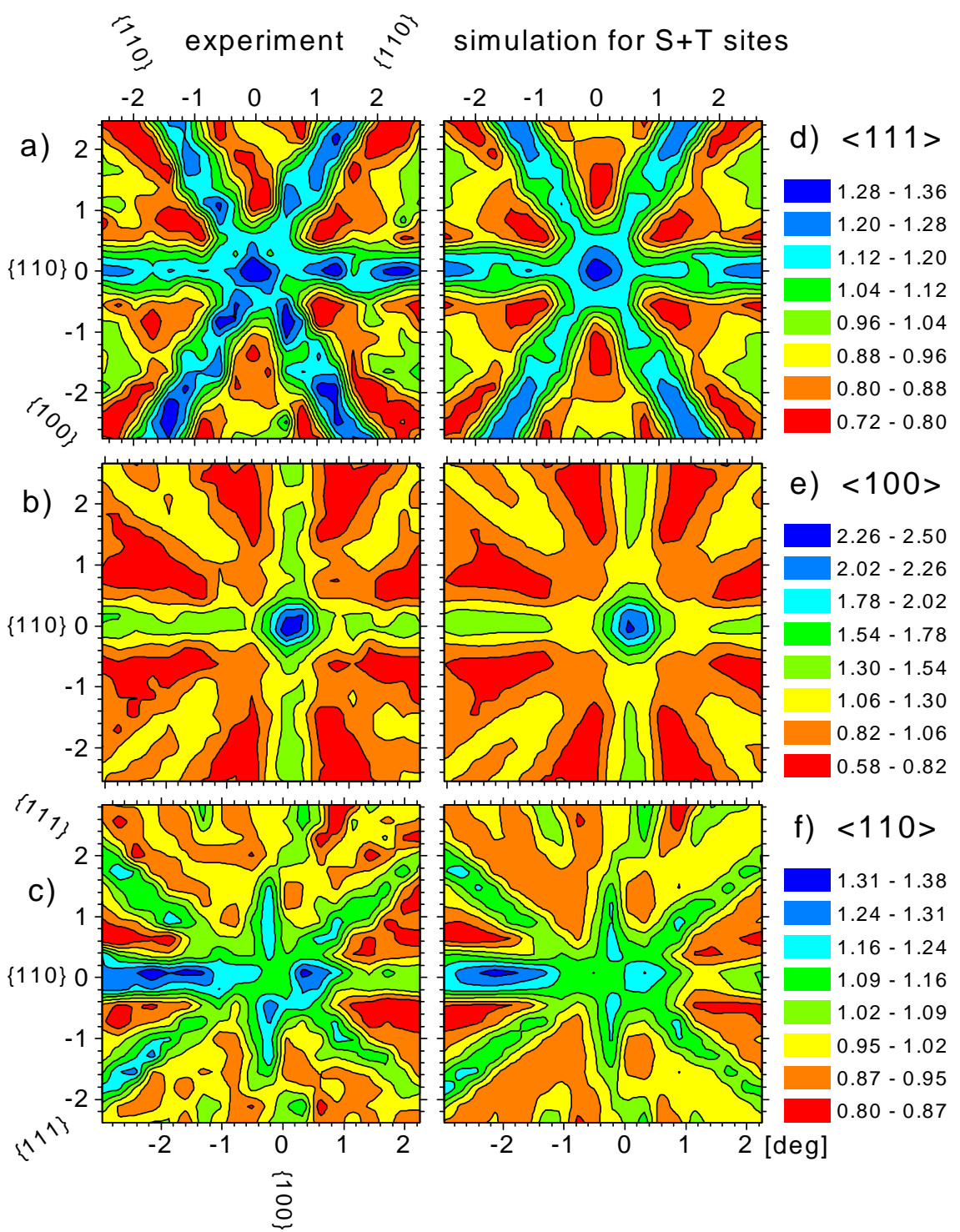

Fig. 1. (a)-(c): Channeling patterns from ${ }^{167 \mathrm{~m}} \mathrm{Er}$ following room temperature implantation of ${ }^{167} \mathrm{Tm}$ into $\mathrm{n}$ GaAs:Te and annealing at $100^{\circ} \mathrm{C}, 200^{\circ} \mathrm{C}$ and $300^{\circ} \mathrm{C}$ for $10 \mathrm{~min}$. (d)-(f): Best fits of simulated patterns to the experimental yields, corresponding to the Er fractions on $\mathrm{S}_{\mathrm{Ga}}$ and $\mathrm{T}_{\mathrm{As}}$ sites given in the text. 


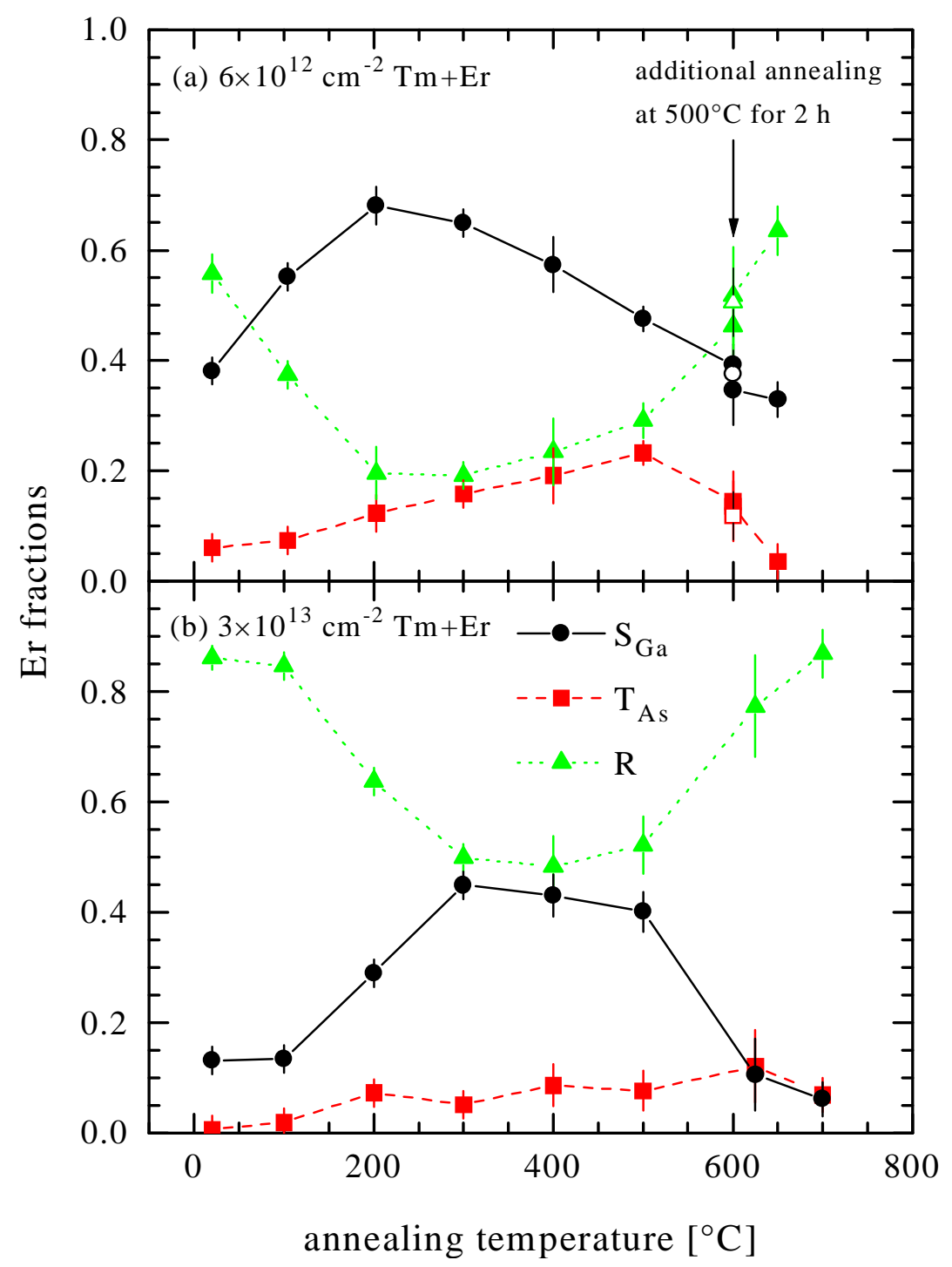

Fig. 2. Fractions of ${ }^{167 \mathrm{~m}} \mathrm{Er}$ on $\mathrm{S}_{\mathrm{Ga}}, \mathrm{T}_{\mathrm{As}}$ and $\mathrm{R}$ sites as function of isochronal (10 min) annealing temperature for samples A (a) and B (b). All measurements were done at room temperature, except the open data points for sample A, which were measured at $500^{\circ} \mathrm{C}$ following the $600^{\circ} \mathrm{C}$ anneal. 